\title{
Philosophiques
}

\section{Entre figures de la philosophie : réflexion sur les Figures de la philosophie québécoise}

\section{A. Voho Sahi}

Volume 16, numéro 2, automne 1989

URI : https://id.erudit.org/iderudit/027084ar

DOI : https://doi.org/10.7202/027084ar

Aller au sommaire du numéro

Éditeur(s)

Société de philosophie du Québec

ISSN

0316-2923 (imprimé)

1492-1391 (numérique)

Découvrir la revue

Citer ce document

Voho Sahi, A. (1989). Entre figures de la philosophie : réflexion sur les Figures de la philosophie québécoise. Philosophiques, 16(2), 347-358.

https://doi.org/10.7202/027084ar d'utilisation que vous pouvez consulter en ligne.

https://apropos.erudit.org/fr/usagers/politique-dutilisation/ 
PHILOSOPHIQUES, Vol. XVI, Numéro 2, Automne 1989

\title{
ENTRE FIGURES DE LA PHILOSOPHIE : RÉFLEXION SUR LES FIGURES DE LA PHILOSOPHIE QUÉBÉCOISE
}

\author{
par A. Voho Sahi
}

Le Département de philosophie de l'Université du Québec à Montréal vient de publier le deuxième tome des Figures de la philosophie québécoise ". Ce volume est consacré aux " philosophes " québécois du XIXe siècle (après les troubles de 1837) dont il comporte un répertoire bibliographique des plus complets.

Le texte présente l'intérêt de n'être pas seulement une présentation très érudite des protagonistes de l'histoire intellectuelle du Canada français, il a en outre le mérite d'exposer les fondements théoriques du projet de reconstitution d'une "philosophie québécoise ».

L'expression "philosophie québécoise » soulève en effet une difficulté préalable qu'il faut résoudre. Que signifie la philosophie lorsqu'elle se voit adjoindre l'épithète "québécoise »? Avant les Québécois, cette question s'est posée dans des termes analogues aux Africains; elle continue d'alimenter le débat sur l'existence et la nature d'une «philosophie africaine». C'est ce qui explique pourquoi, dès la première page du livre, André Vidricaire, en guise d'introduction ${ }^{2}$, se réfère à ce débat afro-africain (où il prend parti sans le savoir?).

1. C. Auhry, M. Chabot, L. Cournoyer, A. Vidricaire: Figures de la pbilosophie québécoise. UQAM, 1988, série Recherches et théories, collection philosophie québécoise.

2. L'introduction de Vidricaire, "Procédures en histoire de la philosophie québécoise" constitue en fait l'objet principal de notre réflexion. 
L'auteur entend appliquer à la culture québécoise, les «arguments» du philosophe béninois p. Hountondji «sur la philosophie africaine ${ }^{3}$.

«(Les lecteurs) verront, sans doute, dans ce projet de reconstituer une philosophie québécoise une nouvelle ETHNO-PHILOSOPHIE! En effet, comme l'a si bien montré Hountondji pour la philosophie africaine, que peut être une philosophie québécoise qui ne s'appuie pas sur des textes philosophiques, sinon une recherche de la vision du monde d'une collectivité qu'on qualifie après coup de philosophie... » 4 .

Ce passage indique d'emblée la position théorique et l'orientation méthodologique adoptées par les auteurs. Mais le commencement d'une philosophie (même s'il ne s'agit ici que de " procédures en histoire de la philosophie ") possède toujours, comme le notait Hegel, une incommodité foncière en ce que son objet est toujours immédiatement exposé au doute et à la controverse, aussi bien dans son contenu que dans sa formes.

C'est pourquoi nous voudrions, dans cette présentation de l'œuvre des Québécois, interroger le transfert et l'appropriation de méthodes qui fondent le projet dont il émane. Il faut à la fois reconnaître ce transfert là où il se trouve et la fonction qui lui est donnée, en principe, pour voir s'il est possible, en fait, et ainsi être en mesure d'évaluer son rôle apparent et son rôle réel de fondement.

\section{I}

L'adoption de la thèse et des positions de Hountondji par les Québécois est une sorte de preuve par les effets. La thèse de Hountondji, formulée dès 1969 dans sa forme la plus lapidaire est la suivante:

«J'appelle philosophie africaine un ensemble de textes: l'ensemble, précisément, des textes écrits par des Africains et qualifiés par leurs auteurs eux-mêmes de "philosophiques ${ }^{6}$

3. P. Hountondjl: Sur la "philosophie africaine», Paris, Maspero, 1977. Il s'agit de l'édition des conférences et articles de Hountondji sur la philosophie africaine entre 1969 et 1973.

4. Figures, p. 3.

5. Heciel: La science de la logique, trad. Bourgeois, Paris, 1970. Introduction, $\$ 1-3$.

6. P. HounT(ON1)]l, op. cit., p. 11. 
Pour des raisons, au demeurant très diverses, sur lesquelles nous ne pouvons pas nous arrêter ici, la thèse de Hountondji souleva une vaste polémique qui est loin d'être close en Afrique. Signalons tout simplement à titre d'exemple, l'article de Niamkey Koffi, L'impensé de Towa et de Hountondji qui, en 1976, remettait systématiquement en cause "ses fondements et implications idéologiques " ${ }^{7}$.

Il n'en reste pas moins que la position de Hountondji a une valeur que peut-être l'auteur n'a pas vraiment perçue et qu'à notre avis, Vidricaire et les autres révèlent aujourd'hui à travers la notion de «figures». Que signifie en effet cette notion?

Pour répondre à cette question, il faut revenir au titre même de l'œuvre pour nous interroger sur le choix de l'expression «figures de la philosophie québécoise» au détriment d'autres expressions comme "histoire de la philosophie québécoise " par exemple. Ce titre paraît d'autant plus incongru que de fait, les Figures sont une histoire de la philosophie québécoise. L'introduction s'intitule bien "procédures en bistoire de la philosopbie québécoise ${ }^{8}$ et de fait, les intellectuels présentés dans le livre, Médéric Lanctôt (1838-1877), Benjamin Pâquet (1832-1900) etc., ainsi que les textes et les courants de pensée appartiennent à l'histoire du Québec. Et pourtant ce n'est pas en tant que figures philosophiques ou historiques qu'ils prennent place dans le livre mais comme «figures de la philosophie québécoise ».

Il y a ici plus qu'une simple question de vocabulaire ou, plus précisément, le choix et l'ordre des mots indiquent le sens où il faut comprendre le projer et lire le livre qui le réalise. Dans l'expression «figures de la philosophie», le poids du sens est porté par le mot philosophie. L'expression met ici «l'idée de philosophie» au-

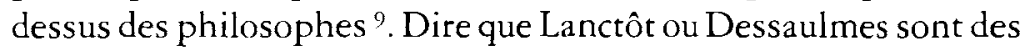

7. Niamkey KOFFi, L'impensé de Towa et de Hountondji, Annales de l'Université d'Abidjan, Série D. (Lettres), tome 9, 1976. Cet article fut publié par la suite dans plusieurs revues internationales.

8. C'est nous qui soulignons.

9. Il y aurait ici un long développement à faire sur la notion de figure, tant il est vrai, comme le notait Michel Guérin, que «la tâche méthodologique qui attend la philosophie esr celle d'une figurologie ». Le génie du philosophe. Paris, Seuil, 1979, p. 48. Mais cela nous écarterait de notre propos qui se veur, après tout le compte rendu d'un livre. 
figures de la philosophie c'est dire qu'ils sont comme des formes où la philosophie prend un contenu dans l'histoire.

Nous sommes ainsi dans une conception phénoménologique de l'histoire de la philosophie. Or c'est cette conception que Hountondji développe au sujet de la philosophie africaine. C'est en ce sens que l'analyse qu'il fait de cette philosophie, de son évolution et de son exercice se révèle comme l'horizon de toute entreprise similaire. Dans une "conférence bégayée » ${ }^{10}$ à l'université de Nairobi le 5 novembre 1973, le philosophe béninois disait :

«Reprenons donc les choses de plus haut. Ce qui est en cause, c'est l'idée de philosophie... Est en cause, en un mot, l'univocité du mot philosophie à travers les diverses applications géographiques.

J'affirme, pour ma part, que cette univocité doit être maintenue. Non pas que la philosophie doive développer les mêmes thèmes ni même poser les mêmes problèmes d'un pays à l'autre, d'un continent à l'autre, mais parce que ces différences de contenu n'ont de sens qu'en tant que différences de contenu, renvoyant, comme telles, à l'unité principielle d'une même discipline, d'un même style d'interrogation " ${ }^{11}$.

Ce texte est sans doute celui qui situe le mieux l'entreprise des Québécois. Il y a, nous dit Hountondji, une « unité principielle d de la philosophie qui ne saurait être remise en cause sous peine de frapper de vanité toute tentative de reconstitution d'une philosophie locale.

La philosophie reste donc au-delà de toutes les philosophies et cependant, chacune de ces dernières n'a de sens que pour autant qu'elle y participe. Les philosophies locales sont donc des manifestations, évidemment incomplètes parce que géographiquement limitées, de cette philosophie «principielle».

Cela veut dire que la philosophie est sans que l'on puisse dire a priori ce qu'elle est. Lorsqu'on se propose de reconstituer la philosophie d'une collectivité, la difficulté « principielle » est donc de trouver les critères permettant de reconnaître le " philosophique » dans la culture de cette collectivité. Puisque la philosophie se donne à voir dans l'espace et dans le temps, il s'agit d'identifier le mode de son apparition.

10. L'expression est de l'auteur, op. cit., p. 51 note.

11. P. Hountondj, op. cit., "L'idée de philosophie ", P. 52-53. 
Pour Hountondji, la philosophie apparaît dans la production littéraire d'individus : la philosophie d'un peuple, c'est l'ensemble des écrits philosophiques d'individus appartenant à ce peuple ${ }^{12}$. Chaque écrit de ce type est un paradigme de l'idée de philosophie, ou, comme le disent les Québécois, une figure de la philosophie.

Les «figures de la philosophie» désignent donc ce que Hountondji appelle les «différences de contenu » c'est-à-dire, les différents contenus que la philosophie prend à telle ou telle époque, chez tel ou tel peuple. Et, comme ces «contenus » n'ont de sens que dans la mesure où ils renvoient à l'unité de la philosophie, il faut dire que ce sens est plus ou moins adéquat suivant qu'en ces contenus la philosophie prend plus ou moins conscience d'ellemême. C'est ce qui explique que pendant longtemps, «les auteurs africains, s'essayant à la philosophie » ont méconnu la réalité de leur propre discours. Celui-ci se prenait pour la métaphilosophie "d'une "philosophie" implicite conçue comme un système de pensée collectif, spontané, irréfléchi, commun à tous les Africains » alors que c'est ce discours lui-même qui est philosophie; son objet n'étant qu' «une invention verbale, un mutbos ${ }^{13}$.

Il y a cependant une différence entre les expressions «différences de contenus» de la philosophie et «figures de la philosophie » québécoise. À ne parler que de différences de contenus on risque de ne faire qu'une histoire de la philosophie en Afrique ou au Québec, c'est-à-dire, examiner la production philosophique de ces collectivités comme parties de la philosophie gréco-méditerranéenne. Ce qui, à l'évidence, ne correspond pas au but visé par Vidricaire et ses collègues qui veulent réaliser une « histoire de la philosophie québécoise »; l'épithète introduisant une connotation anthropologique ou ethnologique.

Si donc le titre adopté trouve sa justification théorique chez Hountondji, il faut dire que les Québécois donnent un contenu plus concret à sa pensée. Une «figure » a le contenu de ce qui y figure c'est-à-dire ici, la philosophie québécoise. La notion de figure

12. Nous nous contentons de restituer l'idéal philosophique de Hountondji sans nuus inrerroger sur ses présupposés nutamment sur le fait qu'il reste tributaire du rationalisme occidental.

13. P. HOUNTONDil, op. cat., p. 52. 
épargne à celle-ci un évanouissement sans reste dans l'histoire de la philosophie sans pour autant l'exposer à l'anathème d'«ethnophilosophie» que les auteurs redoutent. La notion de figure permet en outre la prise en compte de mouvements de pensée ou d'idéologies, comme le libéralisme catholique, dont l'expression écrite constitue en soi une figure dans la mesure où elle se dilue dans d'innombrables articles de journaux dont la plupart sont anonymes ${ }^{14}$.

\section{II}

Mais pourquoi tant de précautions? se demande-t-on. Car après tout, et peut-être avant tout, le Québec n'est pas l'Afrique. Les auteurs des Figures emploient les expressions "canadiensfrançais », pour désigner les philosophes qu'ils étudient, et « Canada français » en parlant du lieu d'émergence de leur discours. Or ces expressions, à moins d'être mises au compte de l'usage, ne renvoient pas à un espace ethnologique au sens où Hountondji entend l'ethnologie.

Selon Hountondji, l'ethnologie comporte un «vice radical» qui a pour nom «ethnocentrisme». L'objet de l'ethnologie est ce dont on parle, un visage sans nom qu'on tente de déchiffrer entre soi, objet à définir et non sujet d'un discours.

«L'ethnologie (ou de quelque autre nom qu'on l'appelle, anthropologie ou comme on voudra) présuppose toujours ce qui est à démontrer : la distinction réelle entre son objet et celui de la sociologie en général, la différence de nature entre les sociétés "primitives" ("archaïques" ou comme on voudra) et les autres sociétés ${ }^{15}$.

Ces lignes ont été écrites à l'époque où on ne parlait pas encore de "l'ethnologie du proche » et où il n'y avait d'ethnologie que du lointain. Or, le philosophe africain constatait avec amertume que «les sociétés qui font l'objet de cette ethnologie là sont toujours des sociétés dominées ». D'où cette conclusion.

"Le discours savant de l'anthropologie n'a de sens qu'à l'intérieur d'un débat scientifique auquel ces peuples n'ont aucune part, mais qui a toujours son origine ailleurs $\gg 16$.

14. "Le libéralisme catholique entre 1868 et 1870 » est l'objet de la dernière partie du livre. Pp. 241 à 307.

15. P. HounTondjl, op. cit., p. 15 et note 4, pp. 15-16

16. Ibid. 
C'est ce constat qui est à l'origine de ce qu'il faut bien appeler le cri d'alarme lancé en 1969 lorsque Hountondji parle de l'ethnophilosophie; ainsi nommée parce qu'elle est un discours "philosophique » élaborée « avant tout pour un public européen» 17. Le terme ethnophilosophie désigne l'«extraversion» (un autre concept cher à Hountondji) ${ }^{18}$ du discours scientifique.

La notion d'ethnophilosophie n'est donc pas strictement liée à l'absence de textes écrits, c'est son origine idéologique qui fait du discours sur la "philosophie africaine » une ethnophilosophie. Ce discours est ethnophilosophie d'abord parce qu'il s'élabore sur et dans le dos de l'Afrique à des fins impérialistes.

S'il en est ainsi, de quelle nature peut être le «possible danger» d'être taxé d'ethnophilosophe que Vidricaire a tenu à rapporter «en noir sur blanc»? 19

En principe, le Québec est une entité ethnologique dans la mesure où il est constitué d'un peuple, qui, à un moment de son histoire s'est trouvé dans une situation de dépendance politique, économique et intellectuelle à l'égard de la France. Le Québec reste par ailleurs une sorte d'enclave culturelle dans le continent nordaméricain, une minorité ethnique.

Une telle situation est toujours propice à l'émergence des porte-parole; ceux qui parlent au nom de... et qui, très souvent sont les mêmes qui parlent de... parce qu'ils se servent de l'objet de leur discours comme tremplin pour exprimer leurs "propres choix théoriques et (leurs) propres options idéologiques ${ }^{20}$. Le Québec peut donc avoir, en principe, ses «québécistes » comme l'Afrique a ses «africanistes».

Mais le danger d'une inversion de la sorte est-il réel, en fait, au Québec? Nous ne saurions le dire. Le fait que Vidricaire en ait conscience témoigne d'une analogie au moins théorique entre l'Afrique et le Canada français.

17. P. HOUNTONDII, op. cit., p. 35.

18. P. HounToNDJI: Situation de l'anthropologue africain: note critique sur une forme d'extraversion scientifique. Communication au colloque interafricain de philosophie, YAMOUSSOUKRO, 13/12/1988.

19. Figures, p. 3.

20. Ibid. 
À la lecture du livre cependant, on ne peut qu'être frappé par la différence de fait entre la situation africaine analysée par Hountondji et celle qu'étudie Vidricaire.

Sur la base des textes répertoriés dans les Figures de la philosopbie québécoise, on peut dire que ni De Calonne, ni Pâquet, etc., qui étaient des prélats n'avaient cherché à reconstituer la vision du monde de Québécois précolombiens comme l'avait fait le père $\mathrm{P}$. Tempeis pour les Bantous du Congo ${ }^{21}$.

"Aux prises avec des problèmes d'ordre politique, économique, culturel et religieux, (les intellectuels québécois du XIX ${ }^{\mathrm{e}}$ siècle) ont fait de Lamennais, Montalembert, Dupanloup, Veuillot, Greely, leurs maîtres à penser ${ }^{22}$.

Ils ont tout simplement philosophé et c'est ce qui fait qu'on ne saurait se tromper sur le sens de leurs productions. Ils n'ont pas cherché à prouver l'existence d'une philosophie québécoise; ils l'ont faite.

Ce fait devrait mettre à l'abri de toute équivoque l'entreprise du groupe de recherche en philosophie québécoise auquel appartient Vidricaire. Il n'a qu'à répertorier cette philosophie faite en laissant de côté celle qui pourrait exister sous le mode vécu et non pensé et dont ses adeptes n'auraient tout au plus qu'une conscience confuse.

Dans ces conditions, il faut prendre les «procédures en histoire de la philosophie québécoise » et la référence à Hountondji comme une résolution tactique et pédagogique. Il s'agit de délimiter l'espace d'investigation pour mieux l'appréhender.

C'est pourquoi il n'était pas nécessaire à Vidricaire de tenir compte des conditions historiques et du contexte intellectuel où émergea la thèse de Hountondji. Il y découvre une vérité dont il se sert comme principe. Et comme, selon le mot de Berkeley, «la vérité peut être découverte brusquement mais doit être enseignée en douceur ${ }^{23}$, Vidricaire et ses collègues laissent le soin à leur

21. Placide Tempels : La philosophie bantoue, Paris, Présence africaine, 1949. Ce livre est à l'origine et au cœur du débat sur la philosophie africaine, Tempels étant considéré comme le père de l'ethnophilosophie africaine.

22. Figures, p. 4.

23. Cité par G. BRYKMAN : «Du commencement introuvable de l'immatérialisme » in, Les Étuder philosophiques, oct. déc. 1980, p. 391. 
livre de révéler dans le détail, progressivement, auteur après auteur, texte après texte, la manière dont ils entendent appliquer les principes de Hountondji dans l'élaboration de l'histoire de la philosophie québécoise.

\section{III}

Deux principes fondamentaux posés par Hountondji dans une conférence donnée à Lumunbachi le 2 juin 1973 fondent la méthode adoptée par les Québécois.

\section{Premier principe}

"Aucune philosophie, si neuve soit-elle, ne naît ex nibilo (...) toute doctrine, en ce domaine, se donne pour une réplique à des doctrines antérieures, sur le mode ambigu de la confirmation et de la réfutation (...) Toute philosophie renvoie, par-devant comme par-derrière, à l'histoire inépuisable de la discipline » ${ }^{24}$.

\section{Deuxième principe :}

"Il n'y a et ne peut y avoir, dans le pur domaine de la pensée, aucune mutation ou révolution, aucun événement au sens fort, qui ne renvoie à quelque événement du monde matériel, et ne doive précisément à ce renvoi d'apparaître lui-même comme un événement ${ }^{25}$.

Ces deux principes fondent ce que Vidricaire appelle les «éléments qui entrent dans la formation d'un système de pensée » 26 . Ils permettent l'élaboration d'une méthode précise pour l'étude et l'exploitation systématiques des textes philosophiques québécois.

Peut-être parce que les Québécois se situent d'emblée pardelà toute polémique sur la nature de la philosophie qu'ils étudient, ils tirent de ces principes ${ }^{27}$, une grille d'interprétation qui permet de comprendre leur propre recherche mais qui peut avoir une valeur tactique et méthodologique pour l'étude de la philosophie africaine qui, il faut le dire, en dépit d'une littérature abondante à son sujet n'est pas encore entreprise.

24. P. Hountundil, op. cit., p. 109.

25. Ibid., P. 113

26. Figures, p. 31.

27. Nous ne sommes pas en mesure de dire si Vidricaire possède l'article ou le texte de la conférence " la philosophie et ses révolutions " d'où sont extraites les citations 
La grille se présente sous la forme d'un triangle isocèle dont la base est constituée par les conditions d'émergence d'un système de pensée, la hauteur par le mode d'appropriation ou d'affranchissement de ces conditions, le sommet étant le système de pensée lui-même. La base fait l'objet d'une analyse de «type externaliste», les éléments représentés sur les côtés et la hauteur et au sommet n'étant perceptibles que dans les textes analysés, font l'objet d'une analyse de «type internaliste ». Ce qui donne le tableau suivant : 28

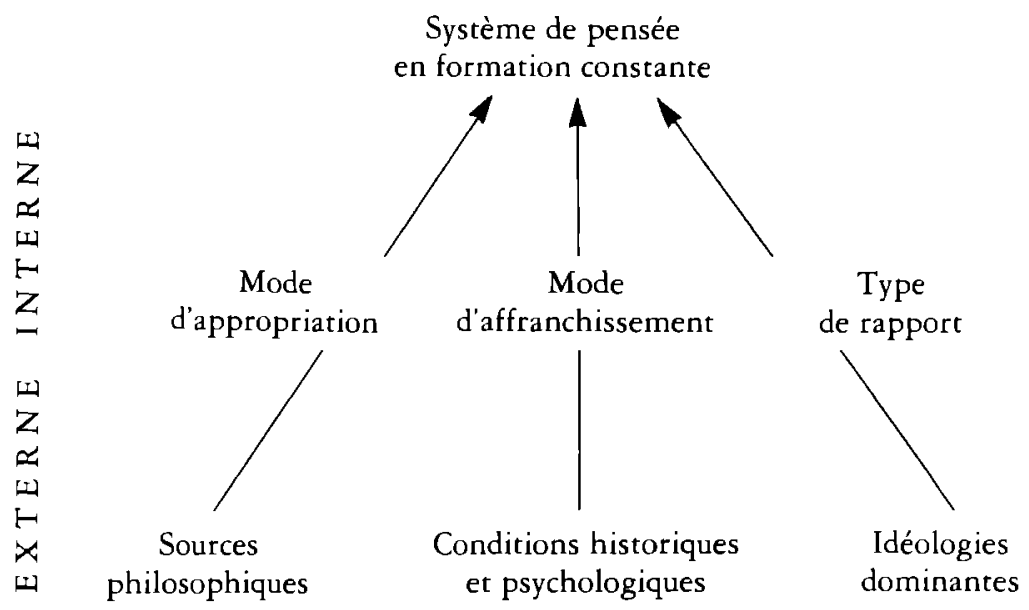

Pour leur part, les auteurs des Figures appliquent strictement cette grille. Concernant les sources philosophiques européennes et américaines, nous apprenons que les philosophes du Québec de la seconde moitié du XIX ${ }^{\mathrm{e}}$ siècle, compte tenu de leur formation et de leur statut de clerc s'inspirent essentiellement des docteurs métropolitains de l'Église comme Lamennais. Quant aux rapports de la pensée avec les conditions historiques, la philosophie de cette époque est marquée par la lutte ouvrière où l'engagement politique d'un Médéric Lanctôt trouve son inspiration. Les idéologies dominantes de cette époque sont l'ultramontanisme et le libéralisme catholique.

28. Ce tableau a été quelque peu simplifié : cf. p. 32 des Figures. 
On pourrait continuer de la sorte à répertorier très aisément tous les autres éléments qui entrent dans les pensées des philosophes québécois. Cet aspect méthodique et pratique des Figures est de loin ce qui donne son prix au livre. Er, pour autant que cette œuvre trouve sa base théorique dans les réflexions en cours sur la philosophie africaine, c'est cet aspect qui lui confère, pour nous, une valeur d'exemple.

On peut toutefois regretter que le souci de suivre (à la lettre?) la thèse de Hountondji ait conduit les auteurs à rejeter, par principe, l'examen de la tradition orale. Compte tenu de ce que nous venons de dire au sujet de l'ethnophilosophie, nous pensons que la considération de la culture orale n'est pas superflue dans la reconstitution de la philosophie d'une collectivité.

En effet, comme l'a montré Kagame pour la philosophie africaine (Rwandaise) ${ }^{29}$ et Heidegger pour la philosophie occidentale ${ }^{30}$, il y a un lien fondamental entre la philosophie et la langue à travers laquelle cette philosophie se fait. C'est dans la tradition orale que les structures de la langue se figent dans les proverbes, les sentences ou les contes.

De ce point de vue, si comme Vidricaire le reconnaît luimême, il est urgent et à propos de se livrer à une recherche sur le statut et la fonction des formes discursives de la culture orale ${ }^{31}$, on ne comprend pas l'exclusion «principielle» de cette forme de culture des Figures de la pbilosophie québécoise.

Les conteurs de la Haute Beauce et de la Mauricie sont peutêtre de grandes figures de la philosophie québécoise et l'urgence et l'à-propos sont, à notre avis, une raison suffisante pour les arracher à l'oubli où l'oralité semble les condamner. Le fait que le Québec, comme l'Afrique, ait ses conteurs à sauver fait que entre les «figures» d'ici et celles de là-bas, la configuration théorique peut s'achever en une tentative commune, celle d'obéir à l'injonction

29. A. KaCiAmE: La philosophie bantu-rwandaise de l'être, Bruxelles, 1956. Ce texte, en dépit des critiques, pour la plupart fondées, que lui fait Hountondji, reste une approche originale et féconde de la philosophie africaine.

30. HerdegGer : Le principe de raison, trad. Preau, Paris, Gallimard, 1962 : cf. Préface de Jean BEAUFRET.

31. Figures, p. 4. 
énigmatique de l'oracle de Delphes : «connais-toi toi-même » qui est la tâche infinie de la philosophie.

Dans cette quête où nous sommes engagés, les auteurs des Figures de la philosophie québécoise viennent de donner aux Africains la preuve de la solidité des principes que nous testons encore. L'adoption des thèses et des principes de Hountondji leur permet de fonder leur entreprise et d'élaborer une méthode d'interprétation de l'histoire de la philosophie.

Même si ce transfert peut comporter quelque malentendu, il montre en tout cas, comme le dirait Descartes, que lorsqu'on ne sait pas comment résoudre une difficulté, il faut adopter un point de vue et s'y tenir. En philosophie québécoise comme en philosophie africaine l'important n'est pas tant la meilleure voie d'accès possible puisque de telle voie il ne peut y avoir. Il faut faire choix d'une voie, la suivre assidûment et méthodiquement, au moins eston sûr d'arriver quelque part.

Département de Philosophie, Faculté des Lettres, Université Nationale de Côte d'Ivoire 